\title{
Lab-on-a-foil devices with integrated retro-reflective structures for multiplexed DNA testing
}

\author{
Pelin Toren ${ }^{1}$ (D) Andreas Proksch ${ }^{1} \cdot$ Martin Smolka $^{1} \cdot$ Anja Haase $^{1}$ - Dieter Nees ${ }^{1} \cdot$ Stephan Ruttloff ${ }^{1}$. \\ Johannes Goetz ${ }^{1}$. Christian Wolf ${ }^{1} \cdot$ Bettina Hierschlager $^{2} \cdot$ Ingo Katzmayr $^{2} \cdot$ Max Sonnleitner $^{2} \cdot$ Barbara Stadlober $^{1}$. \\ Jan Hesse ${ }^{1}$
}

Received: 5 April 2021 / Accepted: 4 May 2021 / Published online: 26 May 2021

(c) The Author(s), under exclusive licence to The Materials Research Society 2021

\begin{abstract}
With the Covid-19-based global pandemic that started in the beginning of 2020, the vital importance of accelerated, reliable and affordable virus testing systems has once again become clearer. Besides, we all learned very well that the disposable biochips, to be used in these in vitro diagnostic (IVD) testing systems, supposed to be produced in large amounts in a very short time to be widely available for the use of humanity to save more and more lives. That is why; roll-to-roll (R2R) polymer structuring manners offer such large quantities for the production of in vitro biochips. Our technology, based on R2R UV nanoimprint lithography (UV-NIL), has superior features. Via our pilot line, robust 7500 biochip components per 100 meter of a flexible, polymer foil coated with a UV curable photo-resin (i.e., parts with capillary fluidic channels or optical structures for IVDs) can be generated. This study shows an example of a prototype of a R2R UV-NIL generated chip: a foil, capillary flow-based IVD biochip for multiplexed DNA detection purposes (i.e., a Lab-on-a-Foil device). The biochip performance was further increased dramatically by integrating UV-NIL produced retro-reflective microstructures, which reflects the light back, to its design to enhance optical signal detection in a commercial IVD device, detecting DNA on a chemiluminescent-reaction basis.
\end{abstract}

\section{Introduction}

Our R2R large-scale polymer imprinting technology provides parallel production of micro- or nano- structures on flexible polymer foils in a high-throughput manner (e.g., 7500 IVD biochip parts either bottom or top, per 100 meter) [1-6]. The process, which is performed at room temperature and ambient pressure, is based on UV-NIL structuring of a positive photo-resin, which is afore coated onto a polymer foil in the beginning of the process line. Our R2R UV-NIL pilot line was shown in Fig. 1. The flexible, transparent polymer foils can be rapidly structured via our imprinting

Pelin Toren and Andreas Proksch have contributed equally to this work.

Pelin Toren

pelin.toerenoezguen@joanneum.at

1 Materials-Institute for Surface Technologies and Photonics, JOANNEUM RESEARCH Forschungsgesellschaft mbH, 8160 Weiz, Austria

2 GENSPEED Biotech GmbH, 4261 Rainbach, Austria technology in multifarious designs (e.g., capillary-based fluidic channels or pumps, optical structures for IVD chips) with various length scales. Using the foils with typical thickness of $\sim 100 \mu \mathrm{m}$, obtaining robust structures with an aspect ratio up to 5:1 (depth: width) [2] is possible.

Therefore, our technology offers remarkable advantages, such as low cost, easy handling and high-throughput manufacturing for polymer-based and disposable in vitro diagnostic biochip production. In order to prove the performance of foil-based IVD chips, a model pathogen testing system with chemiluminescence-based target DNA detection was utilized in this study. A prototype foil DNA chip [1] was batch produced and integration of UV-NIL pyramidal retro-reflective (so-called RR) microstructures was done to investigate the performance of out-coupled light-enhancements in the presence of RR structures. 


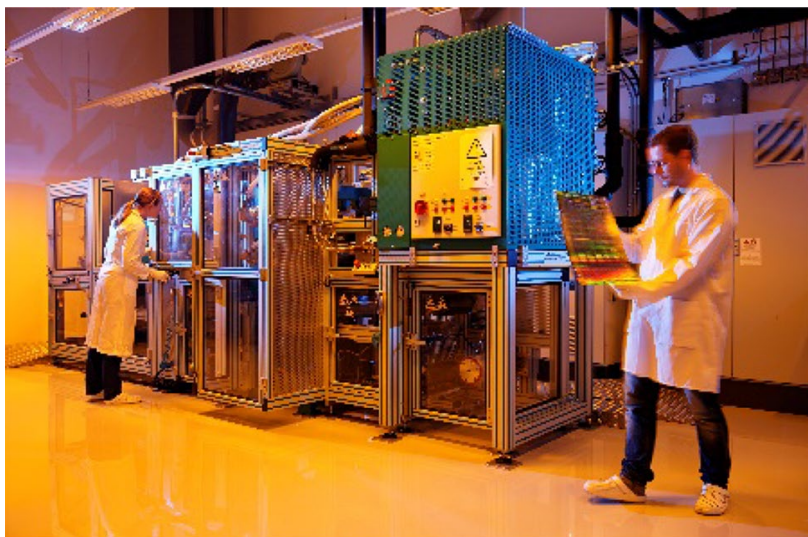

Fig. 1 The photograph of our roll-to-roll (R2R) UV Nanoimprint Lithography (NIL) pilot line

\section{Experimental details}

\section{Production of the foil-chips}

A prototype of our foil-based chip for R2R UV-NIL structuring, which was recently demonstrated in our previous study [1], was produced via a batch imprinting technique. The master structures (i.e., fluidic channels or RR microstructures) were written on a silicon wafer (Si-Mat, Germany) via photolithography. Then the structures were transferred onto a polydimethylsiloxane (PDMS) mold via soft-lithography, allowing 2.5-D topologies in a singlestep production. To produce the PDMS mold, PDMS ${ }^{\circledR}$ Sylgard 184 Silicone Elastomer kit (a PDMS monomer and a curing agent, Sigma-Aldrich, Germany) was utilized. For this purpose, the polymer mould was custommade by mixing the PDMS monomer curing agent with a ratio of $10: 1 \mathrm{w} / \mathrm{w}$, respectively. The mixture was casted over the master and cured on a hotplate for $24 \mathrm{~h}$ at $40{ }^{\circ} \mathrm{C}$, sequentially. The PDMS mould was then detached from the wafer and cut into a final size for UV-NIL structuring purposes. The fluidic structures were transferred to a custom-developed photo-resin (NILcure JR21, Joanneum Research, Austria) coated on a polystyrene (PS) foil with thickness of $190 \mu \mathrm{m}$ (4titude, Germany) by stamping. The curing of the photoresin with the imprinted structures was done using a UV-lamp (365 nm, Waldmann, Germany). To the upper chip parts, a glue was screen-printed prior to the chip bonding with the lower part [1]. The bottom chip part was a PS foil with probe DNA stripes printed using a capillary printer (BioDot ${ }^{\mathrm{TM}}$ Inc., USA). The DNA printing buffer contained Brilliant Blue G (Sigma-Aldrich, Germany) solution and GENSPEED printing buffer with probe ss-DNA concentration of $2 \mu \mathrm{M}$ for each. The chip lamination was performed by combining the two parts
Table 1 Experimental protocol of the foil-chips tested in GENSPEED ${ }^{\circledR}$ R1 device (GENSPEED Biotech, Austria).

\begin{tabular}{llll}
\hline Reagent introduction steps & $\begin{array}{l}\text { Injec- } \\
\text { tion } \\
\text { time }(\mathrm{s})\end{array}$ & Volume $(\mu \mathrm{l})$ & Total volume \\
\hline I. Hybridization solution & 0 & 10 & 20 \\
II. Enzyme solution & 60 & 10 & \\
& 180 & 10 & 30 \\
& 210 & 10 & \\
III. Wash solution & 240 & 10 & \\
& 300 & 10 & 40 \\
& 330 & 10 & \\
IV. Chemiluminescent solu- & 420 & 10 & 20 \\
tion & 450 & 10 & \\
\hline
\end{tabular}

using an office laminator. The UV-NIL structured RR microstructures were integrated at the upper part of the foil chip after bonding. A retro-reflective band (Reflexite ${ }^{\circledR}$ Visibly Better ${ }^{\mathrm{TM}}$ ) was used as master for the RR structures and was transferred to a PDMS mold. This mold was transferred to the top side of the chips by UV imprinting using the custom-developed photoresin NILcure JR21. Another custom-developed photoresin (mr-NIL500SF, micro resist technology, Germany) was utilized as an adhesion promoter. The structures were placed such that they serve as back-reflectors of the incident light generated via a chemiluminescent reaction in the chip. Therefore, a larger portion of the chemiluminescent light was guided towards the photodiodes increasing the signal collection efficiency.

\section{The measurement protocol}

The pipetting protocol for the foil-chips, is provided in Table 1. The chip performance testing was performed using a commercial IVD device: GENSPEED ${ }^{\circledR} \mathrm{R} 1$, which provides rapid testing of hospital-acquired, methicillin-resistant Staphylococcus aureus (MRSA). The measurements were conducted at room temperature using GENSPEED ${ }^{\circledR}$ MRSA hybridization solution which gives positive signals for mecA, SA, PC and $\mathrm{HC}$ genes and negative signals for $\mathrm{SE}, \mathrm{NC}$ and mecC genes. The concentration of all target ss-DNAs was $1 \mathrm{pmol} / \mu \mathrm{l}$ for each (except for HC targets) in the GENSPEED ${ }^{\circledR}$ hybridization solution. All full complementary DNA strands are 25 base-pair long expect for HC, which is 105 base-pair long. The detection is based on DNA hybridization between surface bound ss-DNAs and biotinylated target ss-DNAs in the hybridization solution. The biotin is recognized by a streptavidin-horse radish peroxidase conjugate (SA-HRP) (i.e., commercial GENSPEED ${ }^{\circledR}$ 
enzyme solution). The excess of unbound molecules were removed at the washing step.

The solutions were and the testing procedure was described in detail, in our previous study [1]. The chemiluminescent light is then generated over the DNA-DNA- and biotin-SA-HRP-conjugation via chemiluminescent solution. All solutions are introduced manually into the chip inlet. The readings were done via the GENSPEED ${ }^{\circledR} \mathrm{R} 1$ software. The generated light is detected by a photodiode array composed of 32 photodiodes (i.e., pixels), each with a size of $0.89 \mathrm{~mm} \times 4.39 \mathrm{~mm}$. The pixel distance is $0.1 \mathrm{~mm}$ in the photodiode array.

\section{Data analysis}

Using a custom-developed MATLAB ${ }^{\circledR}$ (MathWorks, USA) tool, the background subtracted net signal (a.u.) was calculated for each corresponding pixel location for printed ss-DNA stripes (the blue lines shown in Fig. 2-(4)) on the foil-chip. The integrated net signal (a.u.) was calculated by considering neighboring pixel (right and left) signals of each main pixel. Each data were provided as a mean value \pm its standard deviation.

\section{Discussion}

The geometry of the UV-NIL produced retro-reflective structures at the upper side of the top biochip part (shown in Fig. 2-(1) and (2), the red zone on the chip) is shown in Fig. 3. The retro-reflective microstructures had a triangle edge and a height of $166 \mu \mathrm{m}$ and $100 \mu \mathrm{m}$, respectively. In order to test the foil-chip performance, two types of chips were used (i.e., foil-chips with and without retro-reflective

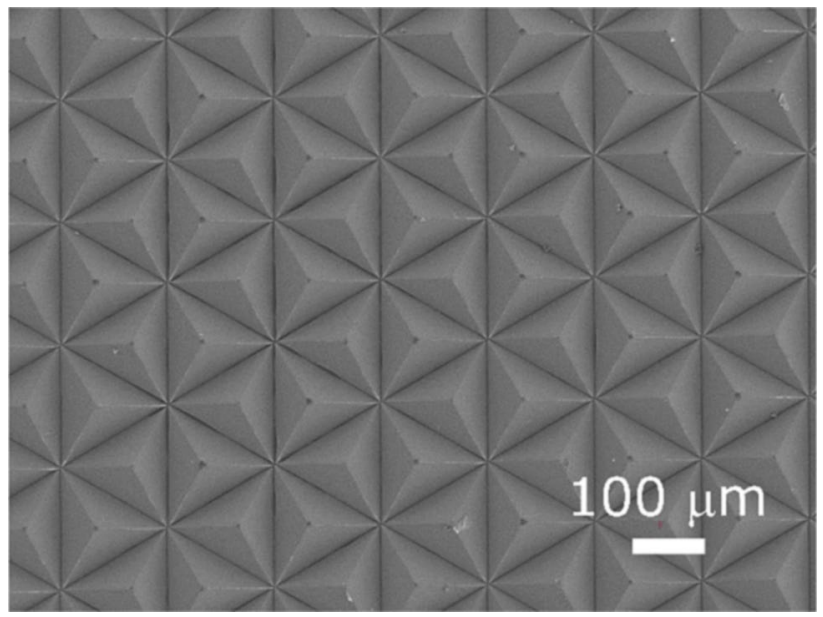

Fig. 3 A scanning electron microscopy (SEM) image of the UV-NIL produced retro-reflective structures

microstructures). The GENSPEED ${ }^{\circledR} \mathrm{R} 1$ readings were analyzed via the MATLAB ${ }^{\circledR}$ tool and the result is provided in Fig. 4. As can be seen in Fig. 4, the modified foil-chips with the RR structures showed higher positive signals (a.u.) as compared to the unmodified foil-chips. The mecA net signal (a.u.), shown as yellow columns in Fig. 4, was dramatically enhanced by a factor of 2.7 in the presence of the RR structures. Likewise, signal enhancements were observed on also SA, PC and HC pixels (Fig. 4, purple, gray and pink columns, respectively). SA, $\mathrm{PC}$ an $\mathrm{HC}$ signals (a.u.) were enhanced in average 1.7-, 1.8- and 2.0folds, respectively, as compared to plain foil chips, in the presence of RR micro-structures. All chip showed low net signals (a.u.) for the negative controls (i.e., SE, NC and mecC pixels), which were below the detection limit (500 a.u. as net signal), as expected.

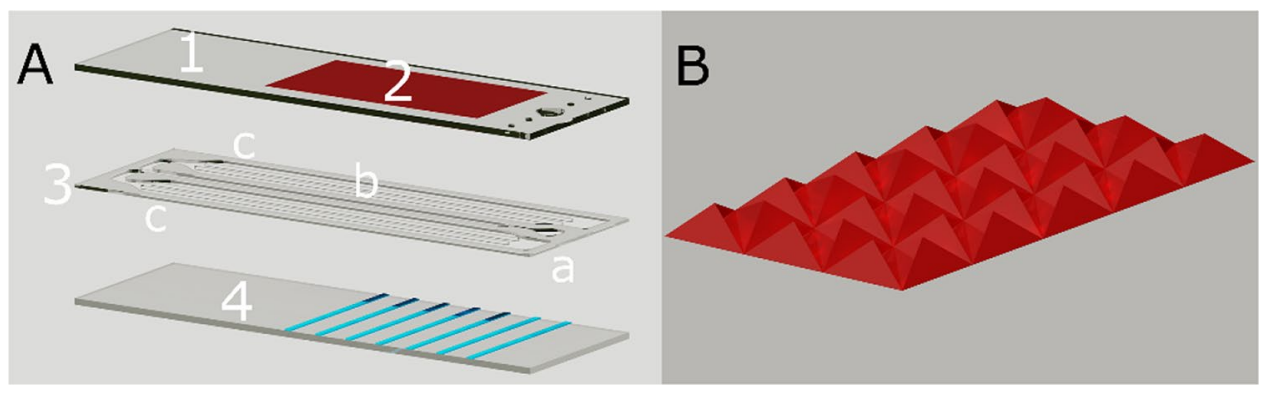

Fig. 2 The illustration of our UV-NIL produced polymer foil-based DNA chip. The chip is composed of two polymer components. a The upper side of the top chip part. (1) The area of UV-NIL structured retro-reflective micro-structures. (2) The lower side of the top chip part, structured with capillary-based fluidic channels composed of (a) retarding ( $5 \mathrm{~mm}$ length, $200 \mu \mathrm{m}$ width, $70 \mu \mathrm{m}$ height), (b) reaction (37 mm length, $2.7 \mathrm{~mm}$ width, $70 \mu \mathrm{m}$ height) and (c) waste chan- nels (total chip height $100 \mu \mathrm{m}$ ). (3) The bottom chip part, at which seven different single-stranded DNAs (i.e., the probes) were printed for a multiplexed DNA detection (shown as blue stripes). (4) The inlet and the small ventilation holes shown both on (1) and (3) were laser drilled. The top chip part was screen-printed using a glue prior to lamination. b The illustration of pyramidal, retro-reflective microstructures in (a)-(2) 


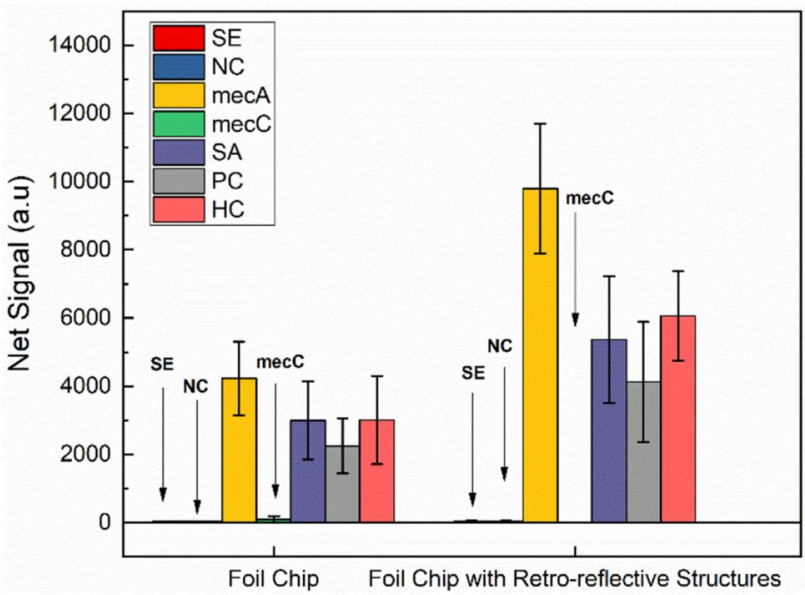

Fig. 4 The results of the UV-NIL structured foil R2R UV-NIL prototypes, obtained using GENSPEED ${ }^{\circledR} \mathrm{R} 1$ reader. Two types of DNA foil chips were generated (i.e., the chips with and without RR microstructures). For each chip type, nine replicates were measured at room temperature. The hybridization solution contained $1 \mathrm{pmol} / \mu \mathrm{l}$ of $\mathrm{mecA}$ targets for the MRSA detection. The response from each DNA stripe of the foil chip (SE, NC, mecA, mecC, SA, PC and HC) was shown with a differently colored column. The data were shown as a mean value \pm standard deviation for each. $\mathrm{SE}, \mathrm{NC}$ and mecC were the negative control signals

\section{Conclusions}

This study shows for the first time retro-reflective microstructure integration into our R2R UV-NIL prototype, foil-based fluidic chip for multiplexed DNA detection purposes [1]. The experimental results revealed that the RR micro-structures on top of the fluidic biochips, significantly enhanced the optical signal collection efficiency and thus the signal at the detectors. Thereby, our results showed the applicability of our foil-based DNA chips, which give distinguishable signals for multiplexed detections, clearly using the commercial GENSPEED ${ }^{\circledR}$ R1 IVD product.

Acknowledgments This project has received funding from the European Union's Horizon2020 research and innovation programme under Grant Agreements with No. 646260 (www.r2r-biofluidics.eu), No. 862092 (www.nextgenmicrofluidics.eu) and No. 871345 (www. medphab.eu). The authors thank to Maria Belegratis, Markus Postl
(JOANNEUM RESEARCH-Materials, Austria) for the support during scanning electron microscopy and the biochip illustration, respectively. We further thank Matija Strbac and Goran Bijelic (Tecnalia, Serbia) for providing the glue printed chip parts. We also thank Mirko Lohse and Manuel W. Thesen (micro resist technology, Germany) for providing the mr-NIL500SFXP photoresin. Additionally, the authors thank to Claude Leiner (JOANNEUM RESEARCH-Materials, Austria) for the pre-investigation of the optical behaviour of the foil chip by optical simulations. PT specially thanks Jan Kafka (Inmold A/S, Denmark) for critically reading the manuscript.

Data availability Due to confidentiality agreements, supporting data can only be made available to bona fide researchers subject to a nondisclosure agreement. Details of the data and how to request access are available from the corresponding author of this article.

\section{Declarations}

Conflict of interest The authors declare that they have no conflict of interest.

\section{References}

1. P. Toren, M. Smolka, A. Haase, U. Palfinger, D. Nees, S. Ruttloff, L. Kuna, C. Schaude, S. Jauk, M. Rumpler, B. Hierschlager, I. Katzmayr, M. Sonnleitner, M.W. Thesen, M. Lohse, M. Horn, W. Weigel, M. Strbac, G. Bijelic, S. Hemanth, N. Okulova, J. Kafka, S. Kostler, B. Stadlober, J. Hesse, Lab Chip 20, 4106-4117 (2020)

2. P. Toren, M. Rumpler, M. Smolka, A. Haase, S. Ruttloff, D. Nees, B. Stadlober, I. Katzmayr, B. Hierschlager, S. Kierstein, M. Sonnleitner, G. Bared, M. Horn, W. Weigel, J. Hesse, Eur. J. Mater. Sci. Eng. 5, 11-16 (2020)

3. B. Stadlober, C. Leiner, D. Nees, A. Haase, U. Palfinger, L. Kuna, S. Ruttloff, M. Smolka, J. Hesse, M. Sonnleitner, C. Sommer, R2R-UV-nanoimprinting as a powerful mean for large-area fabrication of freeform micro-optical elements, Optical Design and Fabrication 2019 (Freeform, OFT), OSA Technical Digest (Optical Society of America), paper OT1A.7 (2019)

4. M. Smolka, S. Ruttloff, D. Nees, C. Prietl et al., High throughput roll-to-roll production of microfluidic chips. Proc. Eur. 2(13), $1054(2018)$

5. M. Leitgeb, D. Nees, S. Ruttloff, U. Palfinger, J. Götz, R. Liska, M.R. Belegratis, B. Stadlober, Multilength scale patterning of functional layers by roll-to-roll ultraviolet-light-assisted nanoimprint lithography. ACS Nano 10(5), 4926-4941 (2016)

6. D. Nees, S. Ruttloff, U. Palfinger, B. Stadlober, Experiments towards Establishing of Design Rules for R2R-UV-NIL with Polymer Working Shims. Proc. SPIE 9777 Alternative Lithographic Technologies VIII, 97770D (2016) 